\title{
BMJ Open A study protocol for a phase II randomised, double-blind, placebo- controlled trial of sodium selenate as a disease-modifying treatment for behavioural variant frontotemporal dementia
}

Lucy Vivash (D) , ${ }^{1,2,3,4}$ Charles B Malpas, ${ }^{1,2,3,5}$ Leonid Churilov, ${ }^{4}$ Mark Walterfang, ${ }^{6,7}$ Amy Brodtmann, ${ }^{3,8,9,10}$ Olivier Piguet, ${ }^{11,12}$ Rebekah M Ahmed, ${ }^{12,13}$ Ashley I Bush, ${ }^{8,10}$ Christopher M Hovens, ${ }^{14}$ T Kalincik, ${ }^{3,5}$ David Darby, ${ }^{1,2,3,4,9}$ Dennis Velakoulis, ${ }^{6,7}$ Terence J O'Brien ${ }^{1,2,3,4}$

To cite: Vivash L, Malpas CB, Churilov L, et al. A study protocol for a phase II randomised, doubleblind, placebo-controlled trial of sodium selenate as a disease-modifying treatment for behavioural variant frontotemporal dementia. BMJ Open 2020;10:e040100. doi:10.1136/ bmjopen-2020-040100

- Prepublication history for this paper is available online To view these files, please visit the journal online (http://dx.doi. org/10.1136/bmjopen-2020040100).

Received 06 May 2020 Revised 07 September 2020 Accepted 26 October 2020

Check for updates

(c) Author(s) (or their employer(s)) 2020. Re-use permitted under CC BY-NC. No commercial re-use. See rights and permissions. Published by BMJ.

For numbered affiliations see end of article.

Correspondence to

Dr Lucy Vivash;

lucy.vivash@monash.edu

\section{ABSTRACT}

Introduction Behavioural variant frontotemporal dementia (bvFTD) is a neurodegenerative disorder often neuropathologically associated with the accumulation of abnormally hyperphosphorylated tau, for which there is currently no disease-modifying treatment. Previous work by our group has shown sodium selenate upregulates the activity of protein phosphatase 2 in the brain, increasing the rate of tau dephosphorylation. The objective of this study is to evaluate the efficacy and safety of sodium selenate as a disease-modifying treatment for bvFTD.

Methods and analysis This will be a multisite, phase $\mathrm{Ilb}$, double-blind placebo-controlled trial of sodium selenate. One hundred and twenty participants will be enrolled across 4 Australian academic hospitals. Following screening eligible participants will be randomised (1:1) to sodium selenate ( $15 \mathrm{mg}$ three times a day) or placebo for 52 weeks. Participants will have regular safety and efficacy visits throughout the study period. The primary study outcome will be percentage brain volume change (PBVC) as measured on MRI over 52 weeks of treatment. This will be analysed with a general linear model (analysis of covariance (ANCOVA)) with the PBVC as an output, the treatment as an input and the baseline brain volume as covariate for adjustment purposes. Secondary outcomes include safety and tolerability measures, and efficacy measures; change in cerebrospinal fluid total-tau, Addenbrooke's Cognitive Examination-III and Cambridge Behavioural Inventory-Revised scores over the 52 weeks of treatment. These will also be analysed with ANCOVA where the corresponding baseline measure will be incorporated in the model. Additional exploratory outcomes will include other imaging, cognitive and biospecimen analyses.

Ethics and dissemination The study was approved by the Human Research and Ethics Committee of the lead site as part of the Australian Multisite Ethics approval system. The results of the study will be presented at national and
Strengths and limitations of this study

- A large placebo-controlled randomised controlled trial of a new drug for behavioural variant frontotemporal dementia (bvFTD).

- Extensive clinical, cognitive and imaging data will be collected producing a highly characterised prospective patient cohort which will be of huge significance to the field.

- A mixture of established and novel diagnostic methods will be used, potentially validating new diagnostic approaches for future use.

- Due to difficulty identifying pathology of bvFTD in life, some participants may not have the pathology targeted by this drug

international conferences and published in peer-reviewed journals.

Trial registration number ACTRN12620000236998.

\section{BACKGROUND}

Behavioural-variant frontotemporal dementia (bvFTD) is the most common clinical syndrome caused by frontotemporal lobar degeneration (FTLD). It is a neurodegenerative disease characterised by the development of progressive behavioural features including disinhibition, apathy, loss of empathy, perseveration and stereotyped behaviour. bvFTD is a devastating condition for both patients and their families, and is invariably fatal. Prevalence rates of bvFTD range from 15 to 50 cases per 100000 people. ${ }^{1-3}$ No approved disease-modifying treatments currently exist for bvFTD, and the few international clinical trials that have been carried out have been 
unsuccessful. ${ }^{4}$ A major unmet need therefore exists in the treatment of this condition. Approximately $45 \%$ of bvFTD cases are classified as 'tauopathies', neuropathologically characterised by abnormally hyperphosphorylated inclusions of the microtubule-associated protein tau. $^{5}$ Hyperphosphorylated tau dissociates from microtubules and aggregates into filamentous tau deposits, disrupting axonal structure and transport leading to neurodegeneration. Targeting this hyperphosphorylated tau is a promising treatment strategy for these patients that requires validation with randomised clinical trials. ${ }^{6}$

\section{Targeting hyperphosphorylated tau}

Two broad approaches are possible to pharmacologically reduce tau hyperphosphorylation in the brain: 1) inhibition of tau serine/threonine kinases or 2) activation of tau serine/threonine phosphatases. Multiple kinases have been implicated in the phosphorylation of tau, hence a more feasible strategy is the upregulation of protein phosphatase 2 (PP2A), the major tau serine/threonine phosphatase present in the brain, which accounts for over $70 \%$ of phosphatase activity. ${ }^{7} \mathrm{PP} 2 \mathrm{~A}$ is co-localised with tau, ${ }^{8-10}$ and reductions in PP2A activity, and therefore reduced tau dephosphorylation are observed in many neurodegenerative diseases. Furthermore, PP2A activity negatively correlates with tau levels in human tissue. ${ }^{10}$

Selenium is an essential trace element in humans and is present in low concentrations in food and the environment. While numerous studies have reported potential chemopreventive benefits associated with selenium dietary supplementation, the therapeutic potential of other selenium compounds has not been widely explored. A large body of preclinical, and emerging clinical data, have provided evidence for the potential of sodium selenate, an oxidised salt of selenium, as a diseasemodifying treatment for hyperphosphorylated tau-based neurodegenerative diseases, traumatic brain injury and epilepsy. ${ }^{11-21}$ Our research has demonstrated significant therapeutic benefits in preclinical models of cancer, ${ }^{12}$ Alzheimer's disease (AD), ${ }^{1322}$ epilepsy ${ }^{15161821}$ and traumatic brain injury, ${ }^{11819}$ and has shown that these benefits were restricted to the administration of the single selenium species, sodium selenate, and were only beneficial in a therapeutic setting when administered in supranutritional doses. ${ }^{172}$

\section{Clinical trials of sodium selenate for other conditions}

Two clinical trials have demonstrated the safety and tolerability of sodium selenate in patients with diseases other than FTD. First, a phase I study in patients with castrationresistant prostate cancer demonstrated a favourable safety profile of VEL015 (sodium selenate) ${ }^{12}$ This study demonstrated that doses up to $60 \mathrm{mg}$ /day were tolerable in chronic dosing, with dose-limiting toxicity observed at $90 \mathrm{mg} /$ day.

More recently, our group completed and published a phase IIa trial of sodium selenate $(30 \mathrm{mg} /$ day $)$ in 40 patients with mild-to-moderate $\mathrm{AD}$, which confirmed the safety and tolerability of the agent. ${ }^{172}$ This AD study was designed to evaluate safety and tolerability and not treatment efficacy, however MR diffusion tensor imaging (DTI) measures were exploratory outcome measures in this study. Voxel-wise analysis of DTI metrics showed relatively less degeneration in the treatment group compared with placebo, which was widespread, but most prominent in the corpus callosum. ${ }^{17}$ Selenium levels in patients' serum and cerebrospinal fluid (CSF) were higher in the treatment group, which is evidence of penetration of the agent across the blood-brain barrier and into the central nervous system. ${ }^{23}$ In patients treated with $10 \mathrm{mg}$ three times a day of sodium selenate, serum selenium levels increased from $145.4 \pm 28.8 \mu \mathrm{g} / \mathrm{L}$ at baseline to $858.3 \pm 446.1 \mu \mathrm{g} / \mathrm{L}$ at week 24, and CSF selenium levels increased from $1.4 \pm 0.5$ to $20.2 \pm 9.1 \mu \mathrm{g} / \mathrm{L}$, no change in serum or CSF levels was observed in the placebo-treated patients. ${ }^{23}$ In addition, selenium levels were inversely correlated with the degree of decline in cognitive measures over the 6-month treatment period, indicating a relationship between drug exposure and neuroprotective efficacy. ${ }^{23}$

\section{Phase I open-label trial of sodium selenate for bvFTD}

We are currently undertaking a single site, exploratory, phase Ib open-labelled study of sodium selenate as a treatment for patients with bvFTD (https://anzctr.org. $\mathrm{au}$, ACTRN12617001218381). The primary objective is to assess the safety and tolerability of a supranutritional dose of sodium selenate in patients with possible bvFTD. Twelve patients have been recruited, and eight patients have completed the 12-month treatment phase so far. There have been no withdrawals, and adverse effects have been relatively mild and similar to those experienced in the phase IIa $\mathrm{AD}$ trial. ${ }^{17}$

Informed by this experience, we will now conduct a multicentred placebo-controlled randomised controlled trial of sodium selenate as a treatment for bvFTD.

\section{AIMS/OUTCOMES}

The aim of this study is to investigate the efficacy and safety of sodium selenate ( $15 \mathrm{mg}$, three times a day) as a disease-modifying treatment of probable bvFTD.

The primary outcome variable is change in whole-brain volume over 52 weeks of treatment.

The secondary outcome variables (safety) will be rate and severity of adverse events and rate of study withdrawal. Secondary efficacy outcome variables are change in CSF total-tau, change in cognition as measured by the Addenbrooke's Cognitive Examination-III (ACE-III) total score and change in behaviour as measured by the Cambridge Behavioural Inventory-Revised (CBI-R) total score over 52 weeks.

Additional exploratory outcomes will investigate changes in CSF, serum and plasma protein biomarkers (total-tau, phospho-tau and neurofilament light chain $(\mathrm{NfL})$ ), changes in other measures of cognition and behaviour (memory, language, executive function, 
attention) and changes in additional neuroimaging measures, including cortical thickness, volumetrics, advanced MRI (diffusion, quantitative susceptibility mapping (QSM), resting state) and fluoro-deoxy glucose (FDG) and tau-binding positron emission tomography (PET). The study will also use advanced statistical modelling to identify predictors of treatment response and non-response, and correlations between biomarkers and cognitive function and behaviour.

\section{METHODS/DESIGN}

This is a multisite, phase IIb double-blind, randomised, placebo-controlled trial of sodium selenate as a treatment for probable bvFTD. Patients will receive 52 weeks of treatment with either sodium selenate $(15 \mathrm{mg}$ three times a day) or placebo. One hundred twenty patients will be recruited into this study. The study will be conducted at four centres in Melbourne and Sydney. The study is funded by the Australian National Health and Medical Research Council (APP1170276). Ethics approval was granted by the Alfred Health Human Research Ethics Committee, Melbourne (609/19).

\section{Eligibility criteria}

Inclusion criteria: participants will be aged over 35 , have a diagnosis of probable bvFTD ${ }^{24}$ and a modified Hachinski score $<5 .{ }^{25}$ Participants must be using effective contraception for the duration of the trial. Participants must have a lumbar puncture and MRI performed during screening. The structural brain MRI must be consistent with a bvFTD diagnosis with no other gross structural abnormalities indicating another neurological disorder. The participant must have a negative amyloid-PET scan (either as part of screening assessments or historical). The participant must live in the community and have at least 10 contact hours per week with a responsible carer. The carer should be capable of ensuring the participant's compliance with the medication and study, and complete questionnaires about the participant's behaviour throughout the study. Written informed consent must be obtained from the participant or their legally authorised representative (as required by local laws and regulations), and the participant's carer.

Exclusion criteria: participants will be excluded based on history of substance use disorder (including alcohol and cannabis); previous participation in an interventional clinical trial (within 3 months of screening), with the exception of prior exposure to sodium selenate; known sensitivity to selenium, sodium selenate, any medicine or vitamin-containing sodium selenate, similar agents or any of the excipients (including microcrystalline cellulose) used; known history of familial $\mathrm{AD}$ or genetic form of FTD that is not considered a primary tauopathy (eg, $G R N$ mutation or C9ORF72 expansion); lifelong primary psychiatric disorder; significant traumatic brain injury with loss of consciousness $>5 \mathrm{~min}$; significant medical or neurological disease, with the exception of bvFTD that is not adequately controlled by therapy and may interfere with the patient's ability to complete the study or affect the patient's cognitive performance; contraindication to MRI or LP; significant impairment of renal, hepatic or haematological function; participant is or has (within 6 weeks of the screening visit) taken any of the following: NMDA receptor antagonists, oral and/or injectable steroids, digoxin, phenobarbitone or warfarin; commencement or titration of other medications known to have an effect on mood or cognition within the 4 weeks prior to screening, including anticholinergics, hypnotics, sedatives, anxiolytics, antidepressants, antiepileptics, antipsychotics, memory-enhancing drugs, nutraceuticals, and other supplements which contain selenium.

\section{Intervention, randomisation and blinding}

Each participant will be provided a unique screening number on signing the consent form. Once all screening assessments have been completed and the participant is eligible to continue, the participant will be assigned a sequential randomisation number generated within the redcap electronic casereport form (eCRF; subject to entry of key data into the eCRF). This randomisation number will be provided to the unblinded site pharmacist who will dispense the drug/placebo in accordance with the randomisation schedule.

Participants will receive either sodium selenate or placebo (1:1) for 52 weeks. Each capsule will contain $5 \mathrm{mg}$ of drug or placebo. The initial dose will be two capsules $(10 \mathrm{mg})$ three times a day, increasing to three capsules $(15 \mathrm{mg})$ three times a day at week 4 , subject to tolerability. In the event of adverse events potentially related to the study drug administration, treatment may be temporarily interrupted and a single within-subject dose reduction, to $10 \mathrm{mg}$ three times a day will, at the investigator's discretion, be allowed. An additional down-titration to $5 \mathrm{mg}$ three times a day will only be allowed after consultation with the medical monitor.

The study participant, their study partner and all site staff (with the exception of the pharmacy team) will remain blinded throughout the course of the study. Individual unblinding envelopes for emergency unblinding of individual patients will be kept at the study sites. The data safety monitoring board (DSMB) will also be blind to study treatment when reviewing safety data. In the event of unexpected adverse events, the DSMB may request unblinding of data to assess potential causality.

\section{Procedures and assessments}

Participants will undergo study testing and procedures as outlined in table 1. Briefly, at the screening assessment, the participant will be reviewed to ensure they meet all the inclusion criteria and none of the exclusion criteria. Neuroimaging (MRI and amyloid-PET) will follow to strengthen the bvFTD diagnosis (and exclude AD). At the baseline visit, the inclusion and exclusion criteria will be reviewed to ensure the participant remains eligible for the study. The participant will undergo an FDG-PET, 
Table 1 Schedule of assessments

\begin{tabular}{|c|c|c|c|c|c|c|c|c|c|c|c|c|c|}
\hline \multirow[b]{2}{*}{ Visit \# } & \multicolumn{4}{|c|}{ Screening Baseline } & \multirow[b]{2}{*}{ TC1 } & \multirow[b]{2}{*}{ TC2 } & \multirow[b]{2}{*}{ TC3 } & \multirow[b]{2}{*}{3} & \multirow[b]{2}{*}{4} & \multirow[b]{2}{*}{5} & \multirow[b]{2}{*}{6} & \multirow[b]{2}{*}{7} & \multirow[b]{2}{*}{8} \\
\hline & $1 a$ & $1 b$ & $2 a$ & $2 b$ & & & & & & & & & \\
\hline Week & \multicolumn{2}{|c|}{-8 to 0} & -2 & 0 & 2 & 4 & 6 & 8 & 16 & 26 & $\overline{39}$ & 52 & 56 \\
\hline Assess eligibility & \multicolumn{2}{|l|}{$X$} & & & & & & & & & & & \\
\hline Confirmation of eligibility & & & & $x$ & & & & & & & & & \\
\hline Confirmation of Dx of bvFTD & $\mathrm{X}$ & & & & & & & & & & & & \\
\hline MRI scan & $\mathrm{X}$ & & & & & & & & & & & $x$ & \\
\hline Amyloid PET scan & & $x$ & & & & & & & & & & & \\
\hline FDG-PET scan & & & $\mathrm{X}$ & & & & & & & & & $\mathrm{X}$ & \\
\hline tau PET scan (optional) & & & $x$ & & & & & & & & & $\mathrm{X}$ & \\
\hline 12-Lead ECG & $x$ & & & $x$ & & & & $x$ & $x$ & $x$ & $x$ & $\mathrm{X}$ & $x$ \\
\hline Neurological examination & $x$ & & & $x$ & & & & & & $x$ & & $x$ & \\
\hline Modified HIS & $x$ & & & & & & & & & & & & \\
\hline ACE-III & $x$ & & & $x$ & & & & & & $x$ & & $x$ & \\
\hline Cognitive and behavioural battery & & & & $\mathrm{X}$ & & & & & & $x$ & & $\mathrm{X}$ & \\
\hline C-SSRS & $x$ & & & $x$ & & & & $\mathrm{X}$ & $\mathrm{X}$ & $x$ & $x$ & $\mathrm{X}$ & $\mathrm{X}$ \\
\hline Haematology and biochemistry & $x$ & & & $x$ & & & & $x$ & & $x$ & $x$ & $x$ & $x$ \\
\hline Coagulation & $x$ & & & & & & & & & & $x$ & & \\
\hline Redispense drug & & & & & & & & $x$ & & & & & \\
\hline Drugadministration & & & & $x$ & & & & $\mathrm{X}$ & & & & & \\
\hline Dispense diary card & & & & $x$ & & & & $\mathrm{X}$ & $x$ & $\mathrm{X}$ & $x$ & $\mathrm{X}$ & \\
\hline Review diary card & & & & & $x$ & $x$ & $\mathrm{X}$ & $x$ & $x$ & $x$ & $x$ & $x$ & $\mathrm{X}$ \\
\hline Review of AEs/SAEs & & & & $x$ & $\mathrm{x}$ & $x$ & $x$ & $x$ & $x$ & $x$ & $x$ & $x$ & $x$ \\
\hline Review of concomitant medications & $X$ & & & $\mathrm{X}$ & $\mathrm{X}$ & $X$ & $\mathrm{X}$ & $\mathrm{X}$ & $\mathrm{X}$ & $\mathrm{X}$ & $\mathrm{X}$ & $\mathrm{X}$ & $\mathrm{X}$ \\
\hline
\end{tabular}

ACE-III, Addenbrooke's Cognitive Examination-III; AE, adverse event; bvFTD, behavioural variant frontotemporal dementia; C-SSRS, Columbia-suicide severity rating scale; Dx, diagnosis; FDG, fluoro-deoxy glucose; hCG, human chorionic gonadotropin; HIS, Hachinski ischaemic scale; PET, positron emission tomography; SAE, serious adverse event.

baseline CSF sampling and a cognitive and behavioural assessment. Lastly, the participant will be administered the study drug $(10 \mathrm{mg})$ in the clinic and multiple blood draws taken for pharmacokinetic analysis.

Follow-up phone calls will be made to monitor for adverse events. If participants are tolerating the study drug, they will be instructed to increase to three capsules ( $15 \mathrm{mg}$, three times a day) at week 4 . Diary cards will be given to participants to record adverse events that occur between clinic visits.

Participants will attend regular in-clinic study visits to assess their safety and well-being, and to resupply the study medication. The cognitive and behavioural assessment will be repeated at weeks 26 and 52. Prior to their week 52 clinical visit, participants will undergo repeat neuroimaging (MRI, FDG-PET) and CSF sampling for 
biomarker analyses. Participants will attend a final safety follow-up visit 4 weeks following the cessation of the study drug.

\section{Measures}

Neuroimaging

MRIs will be acquired during the screening period and week 52. The MRI protocol includes the following sequences: volumetric T1 $\left(1 \mathrm{~mm}^{3}\right)$, T2-space $(0.8 \times 1.1 \times 4$ $\mathrm{mm})$, volumetric T1 $\left(1 \mathrm{~mm}^{3}\right)$, multiband DWI $\left(2.5 \mathrm{~mm}^{3}\right)$, resting state functional MRI $\left(4.5 \mathrm{~mm}^{3}\right)$, multi-echo QSM $\left(3.5 \mathrm{~mm}^{3}\right)$.

The primary outcome measure will be the change in whole-brain volume (PBVC) from baseline to 52 weeks. Whole-brain volume will be measured using T1-weighted structural MRI acquired at baseline and after 52 weeks of treatment. Change in whole-brain volume will be computed using the SIENA module within the FSL software.$^{26}$ Briefly, SIENA performs brain extraction, halfway-space registration and tissue segmentation. Perpendicular edge displacement is then computed between the brain/non-brain edge points of the two images. The accuracy of this method is very high, with longitudinal error reported at $0.15 \% .{ }^{27}$

Florbetaben PET will be conducted at screening to exclude $\mathrm{AD}$; $300 \mathrm{MBq}( \pm 10 \%)$ will be injected intravenously, 90 min postinjection a dynamic three-dimensional scan $(4 \times 300$ s frames $)$ will be acquired. This will not be required for participants who have a historical negative amyloid PET that is available for review by the site principal investigator.

FDG-PET will be conducted at baseline and week 52 to assess disease progression. $185 \mathrm{MBq}( \pm 10 \%)$ will be injected intravenously, the patient will rest in a quiet room for $20 \mathrm{~min}$, and a dynamic $3 \mathrm{D}$ acquisition will begin 30 min post-injection $(6 \times 300 \mathrm{~s}$ frames $)$.

An optional substudy investigating tau PET as a marker of disease progression will also be conducted. Participants who consent will have two tau PET scans, at baseline and in the 2 weeks prior to week 52. A dynamic 3D acquisition will begin concurrently with the intravenous injection of $185 \mathrm{MBq}( \pm 10 \%)$ of PI-2620.

\section{Cognitive and behavioural battery}

The following cognitive and behavioural scales will be administered at baseline, week 26 and week 52 of the study.

\section{Addenbrooke's Cognitive Examination III}

The ACE-III is a broad cognitive screening test of five cognitive domains: attention/orientation, memory, language, verbal fluency and visuospatial skills, producing a score out of 100 (normal scores $\geq 88 / 100$ ). Administration takes about $15 \mathrm{~min}$ and three different versions are available. ${ }^{28}$ This will be analysed as one of the secondary efficacy end points.

\section{NIH Toolbox}

The NIH Toolbox is a multidimensional battery of measures used to assess cognitive, sensory, motor and emotional function in people. The NIH Toolbox has been validated for those aged 3-85 years and is designed such that repeated assessments can be completed for longitudinal monitoring of disease progression. The NIH Toolbox takes approximately $30 \mathrm{~min}$ to administer. ${ }^{29} 30$

\section{Hayling Sentence Completion Test}

The Hayling Sentence Completion test is a measure of response initiation and response suppression. It is entirely verbal, and therefore can be administered to patients with problems with reading and visual perception. It consists of two sets of 15 sentences each having the last word missing. In the first section, the examiner reads each sentence aloud and the participant has to simply complete the sentences as quickly as possible, yielding a simple measure of response initiation speed. The second part of the Hayling requires participants to complete a sentence with a nonsense ending word, giving measures of response suppression ability and thinking time. It takes approximately $5 \mathrm{~min}$ to administer and yields three different measures of executive functioning which can be considered separately or combined into an overall score. $^{30}$

\section{Victoria Stroop Test}

The Victoria Stroop Test is a measure of executive function commonly used in neuropsychological evaluation. The test uses three conditions that consist in naming the colour of dots, of neutral words and of coloured words printed in incongruent colours. Each condition contains 24 items. Because of its short administration time, approximately $5 \mathrm{~min}$, it is appropriate for use in geriatric populations and with those suffering from dementia. ${ }^{31}$

\section{Controlled Oral Word Association Test}

The Controlled Oral Word Association Test is a verbal fluency test that measures executive function. The participant is asked to name words beginning with a designated letter for $1 \mathrm{~min}$ under certain conditions (eg, no repetitions, no proper nouns, no identical stem words) and this procedure is repeated with three different letters. The whole examination usually takes $5-10$ min. $^{32}$

\section{Trail Making Test $A$ and $B$}

The Trail Making Test is a test of visual attention and task switching. It consists of two parts in which the subject is instructed to connect a set of 25 targets as quickly as possible while still maintaining accuracy. There are two parts to the test: in the first, the targets are all numbers (1, 2, 3 and so on), which the participant must connect sequentially; in the second part, the subject alternates between numbers and letters (1, A, 2, B and so on). The first part of the test is used to primarily measure processing speed, while the second part is a test of executive function. The time to complete the paths are the scores for this assessment. ${ }^{33}$ 


\section{Digit Span Test}

The Digit Span Test is a measure of attention and working memory. Participants are told a sequence of digits (which become progressively longer), which they must repeat back to the examiner. This test is performed forwards and backwards. The score is the maximum length of digits they correctly repeated under the two conditions. ${ }^{34}$

\section{Sydney Language Battery}

The Sydney Language Battery is a test of language at a single word level, which measures naming, repetition, comprehension and semantic association. The same set of 30 multisyllabic nouns (3 or more syllables) are used across the four tasks. Words are ordered with decreasing frequency and are graded into three broad levels of difficulty. ${ }^{35}$

\section{Mini-Social Emotional Assessment}

The Mini-Social Emotional Assessment (mini-SEA) is a cognitive battery designed to assess social and emotional function. The battery contains two components: (a) a faux-pas test assessing theory of mind and (b) a facial emotional recognition test. The neural correlates of the mini-SEA have been established in bvFTD, with significant relationships reported with the medial prefrontal cortex. The mini-SEA takes approximately $30 \mathrm{~min}$ to administer. ${ }^{36}$

\section{Cambridge Behavioural Inventory-Revised}

CBI-R is an informant questionnaire that assesses neuropsychiatric and neurobehavioural symptoms in patients with neurodegenerative disease. ${ }^{18}$ The instrument contains 45 questions assessing the following domains: (a) memory and orientation, (b) everyday skills, (c) self-care, (d) abnormal behaviour, (e) mood, (f) beliefs, (g) eating habits, (h) sleep, (i) stereotypic and motor behaviours and (j) motivation. The instrument takes approximately 10 min to complete. A total score is generated that will be used for this study. ${ }^{37}$ This will be analysed as one of the secondary efficacy end points.

\section{Caregiver Burden Scale}

The Caregiver Burden Scale is a caregiver-completed instrument that assesses the experience of burden in people who care for those with cognitive disorders. ${ }^{38}$ The instrument contains 22 questions and takes approximately $5 \mathrm{~min}$ to complete. A total score is generated that will be used for the present study. ${ }^{38}$

\section{Safety assessments}

A 12-lead ECG and a physical examination will be performed at each clinic visit, haematology, chemistry and urinalysis will be performed at each clinic visit except week 16. Neurological examinations will be carried out at screening, baseline, week 26 and 52. Any clinically significant abnormalities in any of these investigations will be considered adverse events. The diary cards will be reviewed to ascertain any adverse events that occurred between visits, and the participant and their study partner interviewed to confirm adverse events and concomitant therapies.

\section{Blood biomarkers}

Additional blood samples will be collected for pharmacokinetic, biomarker and exploratory analyses. Pharmacokinetic sampling $(6 \mathrm{~mL} / \mathrm{sample})$ will be performed at baseline, week 8 and week 52. A predose (1 hour) sample will be taken, then four additional samples, 0.5, 1, 2, 4 hours after dosing. Sodium selenate levels in plasma will subsequently be measured in these samples for determination of the pharmacokinetic profile.

Exploratory sampling will occur at baseline, week 8, week 26 and week 52. Samples will be taken for plasma and serum at each of these visits. Blood for DNA and RNA will be taken only at baseline. Exploratory analyses will include measurement of proteins associated with neurodegeneration (total-tau, phospho-tau, beta-amyloid ${ }_{42}$, $\mathrm{NfL}$ ) in serum and plasma, testing for genetic mutations associated with bvFTD (MAPT, GRN, C9ORF72 expansion). As yet undetermined additional testing may be performed.

\section{CSF biomarkers}

CSF sampling will take place at baseline (pretreatment) and week 52. Atraumatic needles $(20 \mathrm{G})$ will be used for sampling. CSF $(\sim 20 \mathrm{~mL})$ will be collected in polypropylene tubes $(10 \mathrm{~mL})$ cooled on ice. Samples will be kept on ice until processing, aliquoted into $500 \mu \mathrm{L}$ polypropylene aliquots and stored at $-80^{\circ} \mathrm{C}$. Samples will be analysed for beta-amyloid ${ }_{42}$, total-tau, phospho-tau and NfL protein levels. Additional as yet undetermined testing may also be performed. Change in total-tau will be used as a secondary efficacy outcome measure.

\section{Power and sample size}

All outcomes are predefined prospectively in hierarchical order, meaning that the trial will be declared positive or negative based on primary outcome. Statistical power has thus been determined on the PBVC. Previous studies indicate that the mean annual rate of PBVC in FTD is $3.15 \%(\mathrm{SD}=2.08) .{ }^{39}$ The mean atrophy rate in controls is $0.47 \%$. Slowing the rate of atrophy by $50 \%$ (half-way between the FTD rate and the control rate $=1.81 \%$ ) would represent a clinically meaningful treatment effect. Assuming the SD of the FTD group holds across treatment and placebo arms, this would correspond to a medium effect size (Cohen's $d=0.64$ ). Given a critical two-tailed alpha of 0.05 , recruiting 80 participants (randomised 1:1 into two groups) would yield power of 0.80 , to observe such or larger difference. As a proportion of participants will have a non-tau-based pathology (estimated $\sim 30 \%$ based on previous experience), therefore we anticipate a sample size of 120 will allow for adequate power. Due to the previously demonstrated safety and tolerability, no interim analyses of efficacy, futility or safety are planned. 


\section{Outcomes and statistical overview}

\section{Primary end point}

The primary outcome measure will be the PBVC from baseline to 52 weeks between the treatment and placebo groups. All participants with a post-baseline MRI will be included in the primary analysis. This will be analysed with a general linear model (analysis of covariance (ANCOVA)) with the PBVC as an output, the treatment as an input and the baseline brain volume as covariate for adjustment purposes.

\section{Secondary end points}

All continuous secondary efficacy end points will be reported using descriptive statistics (mean, median, minimum, maximum, SD) by visit. Transformations of these end points (such as change and percentage change) will be summarised similarly.

The analysis of change from baseline to week 52 in CSF total-tau, ACE-III and CBI-R will be performed using a general linear model (ANCOVA), where the corresponding baseline measure will be incorporated as a covariate in the model. Estimates will be obtained from the model of the adjusted mean change (with 95\% confidence limits) in marker for treatment.

\section{Safety and tolerability}

For all categorical outcomes frequency tables (number of subjects and percentage) will be presented by visit, these tables will indicate both the number of subjects in the cohort $(\mathrm{N})$ and the number of observations $(\mathrm{n})$.

Safety and tolerability will be determined by frequency of adverse events (Common Terminology Criteria for Adverse Events score $\geq 3$ ), frequency of down-titration events and frequency of study discontinuation.

\section{Monitoring and data quality}

In accordance with International Council for Harmonisation Good Clinical Practice guidelines, the project manager will carry out source document verification at regular intervals to ensure that the data collected in the eCRF are accurate and reliable. The frequency of monitoring visits will be determined by the rate of subject recruitment.

An independent medical monitor has been appointed to provide safety oversight during the conduct of the study, and to review all safety-related events at 3-month intervals.

The DSMB will consist of the medical monitor, an independent clinician and independent biostatistician. The first DSMB review will be conducted within 2 weeks of the third randomised patient reaching their week 8 visit or within 1 week of two SAEs occurring, whichever is first. Subsequent DSMB reviews will occur every 6 months. Additional DSMB meetings may be called at the request of the medical monitor or site principal investigators in the event of urgent safety concerns. After each review, the medical monitor will make recommendations to the principal investigator based on the safety and tolerability issues.

\section{Ethics and dissemination}

The study has been approved by the Alfred Hospital Human Research Ethics Committee (HREC, 609/19). Results of this study will be disseminated through presentations at national and international conferences and published in peer-reviewed journals. Any amendments to the protocol will be approved by the HREC prior to implementation and subsequently updated on ANZCTR.

\section{Patient and public involvement statement}

Patients were not involved in the study conception or design. All study participants will be provided with a plain English summary of the results of the study at its conclusion (a requirement of Australian ethics committees and stated in the study consent form). Dissemination of results to the wider community will be through open events at our hospitals and Dementia Australia as well as media reports.

\section{Author affiliations}

${ }^{1}$ Department of Neuroscience, Monash University, Melbourne, Victoria, Australia

${ }^{2}$ Department of Neurology, Alfred Hospital, Melbourne, Victoria, Australia

${ }^{3}$ Department of Neurology, Royal Melbourne Hospital, The University of Melbourne, Parkville, Victoria, Australia

${ }^{4}$ Departments of Medicine and Radiology, University of Melbourne, Parkville, Victoria, Australia

${ }^{5}$ CORe, Department of Medicine, University of Melbourne, Parkville, VIC, Australia ${ }^{6}$ Department of Neuropsychiatry, Royal Melbourne Hospital, Melbourne, Victoria, Australia

${ }^{7}$ Melbourne Neuropsychiatry Centre, University of Melbourne, Parkville, Victoria, Australia

${ }^{8}$ Florey Institute for Neuroscience and Mental Health, Melbourne, Victoria, Australia ${ }^{9}$ Eastern Cognitive Disorders Clinic, Monash University, Box Hill, Victoria, Australia

${ }^{10}$ Melbourne Dementia Research Centre, University of Melbourne, Parkville, VIC, Australia

${ }^{11}$ School of Psychology, The University of Sydney, Sydney, New South Wales, Australia

${ }^{12}$ Brain and Mind Centre, The University of Sydney, Sydney, New South Wales, Australia

${ }^{13}$ Memory and Cognition Clinic, Royal Prince Alfred Hospital, Sydney, New South Wales, Australia

${ }^{14}$ Department of Surgery, Royal Melbourne Hospital, The University of Melbourne, Parkville, Victoria, Australia

Contributors LV drafted the manuscript. LV, CM, LC, MW, AB, OP, RMA, AB, CMH, TK, DD, DV and TO contributed to study design. All authors edited the manuscript and approved the final version. (NB This is a protocol paper. There are no data, analyses or results.)

Funding This work is supported by the Australian National Health and Medical Research Council (NHMRC) Medical Research Future Fund grant number APP1170276. OP is supported by an NHMRC Senior Research Fellowship (GNT1103258).

Competing interests All authors report a grant from the NHMRC to support this study. The authors report the following disclosures outside of this study: LV reports personal fees from Biogen Australia. AIB reports personal fees from Collaborative Medicinal Development, and shares in Alterity, Cogstate, Grunbiotics and Mesoblast. $\mathrm{CH}$ reports issued patents US 9415063 and US 8920951. TK reports grants from the University of Melbourne and Biogen Australia; personal fees from Biogen, Roche, Genzyme-Sanofi, Merck, Novartis, WebMD Global, Teva and BioCSL; and nonfinancial support from Biogen, Genzyme-Sanofi and Merck. TOB reports research funding from Biogen, Eisai, UCB, Anavex and Praxis. CM, LC MW, AB, OP, RA, DD and DV have nothing to disclose. 
Patient and public involvement Patients and/or the public were involved in the design, or conduct, or reporting, or dissemination plans of this research. Refer to the 'Methods' section for further details.

Patient consent for publication Not required.

Provenance and peer review Not commissioned; externally peer reviewed.

Open access This is an open access article distributed in accordance with the Creative Commons Attribution Non Commercial (CC BY-NC 4.0) license, which permits others to distribute, remix, adapt, build upon this work non-commercially, and license their derivative works on different terms, provided the original work is properly cited, appropriate credit is given, any changes made indicated, and the use is non-commercial. See: http://creativecommons.org/licenses/by-nc/4.0/.

ORCID iD

Lucy Vivash http://orcid.org/0000-0002-1182-0907

\section{REFERENCES}

1 Kettle M. Module 1: what is frontotemporal dementia? In: Frontotemporal dementia toolkit, 2011: 1-24.

2 Coyle-Gilchrist ITS, Dick KM, Patterson K, et al. Prevalence, characteristics, and survival of frontotemporal lobar degeneration syndromes. Neurology 2016;86:1736-43.

3 Ratnavalli E, Brayne C, Dawson K, et al. The prevalence of frontotemporal dementia. Neurology 2002;58:1615-21.

4 Piguet O, Kumfor F, Hodges J. Diagnosing, monitoring and managing behavioural variant frontotemporal dementia. Med J Aust 2017;207:303-8.

5 Chare L, Hodges JR, Leyton CE, et al. New criteria for frontotemporal dementia syndromes: clinical and pathological diagnostic implications. J Neurol Neurosurg Psychiatry 2014;85:865-70.

6 Hanger DP, Anderton BH, Noble W. Tau phosphorylation: the therapeutic challenge for neurodegenerative disease. Trends $\mathrm{Mol}$ Med 2009:15:112-9.

7 Goedert M, Jakes R, Qi Z, et al. Protein phosphatase 2A is the major enzyme in brain that dephosphorylates tau protein phosphorylated by proline-directed protein kinases or cyclic AMP-dependent protein kinase. J Neurochem 1995;65:2804-7.

8 Arnold SE, Hyman BT, Flory J, et al. The topographical and neuroanatomical distribution of neurofibrillary tangles and neuritic plaques in the cerebral cortex of patients with Alzheimer's disease. Cereb Cortex 1991;1:103-16.

9 Hyman BT, Van Hoesen GW, Damasio AR, et al. Alzheimer's disease: cell-specific pathology isolates the hippocampal formation. Science 1984;225:1168-70.

10 Gong CX, Lidsky T, Wegiel J, et al. Phosphorylation of microtubuleassociated protein tau is regulated by protein phosphatase $2 \mathrm{~A}$ in mammalian brain. Implications for neurofibrillary degeneration in Alzheimer's disease. J Biol Chem 2000;275:5535-44.

11 Brady RD, Grills BL, Romano T, et al. Sodium selenate treatment mitigates reduction of bone volume following traumatic brain injury in rats. J Musculoskelet Neuronal Interact 2016;16:369.

12 Corcoran NM, Hovens CM, Michael M, et al. Open-label, phase I dose-escalation study of sodium selenate, a novel activator of PP2A, in patients with castration-resistant prostate cancer. $\mathrm{Br} J$ Cancer 2010;103:462-8.

13 Corcoran NM, Martin D, Hutter-Paier B, et al. Sodium selenate specifically activates PP2A phosphatase, dephosphorylates tau and reverses memory deficits in an Alzheimer's disease model. J Clin Neurosci 2010;17:1025-33.

14 lqbal K, del C. Alonso A, Chen S, et al. Tau pathology in Alzheimer disease and other tauopathies. Biochimica et Biophysica Acta (BBA) - Molecular Basis of Disease 2005;1739:198-210.

15 Jones NC, Nguyen T, Corcoran NM, et al. Targeting hyperphosphorylated tau with sodium selenate suppresses seizures in rodent models. Neurobiol Dis 2012;45:897-901.
16 Liu S-J, Zheng P, Wright DK, et al. Sodium selenate retards epileptogenesis in acquired epilepsy models reversing changes in protein phosphatase $2 \mathrm{~A}$ and hyperphosphorylated tau. Brain 2016;139:1919-38.

17 Malpas CB, Vivash L, Genc S, et al. A phase ila randomized control trial of VEL015 (Sodium Selenate) in mild-moderate alzheimer's disease. J Alzheimer's Dis 2016:1-10.

18 Shultz SR, Wright DK, Zheng P, et al. Sodium selenate reduces hyperphosphorylated tau and improves outcomes after traumatic brain injury. Brain 2015;138:1297-313.

19 Tan XL, Wright DK, Liu S, et al. Sodium selenate, a protein phosphatase $2 \mathrm{~A}$ activator, mitigates hyperphosphorylated tau and improves repeated mild traumatic brain injury outcomes. Neuropharmacology 2016;108:382-93.

20 Whitehouse PJ, Price DL, Struble RG, et al. Alzheimer's disease and senile dementia: loss of neurons in the basal forebrain. Science 1982;215:1237-9.

21 Zheng P, Shultz SR, Hovens CM, et al. Hyperphosphorylated tau is implicated in acquired epilepsy and neuropsychiatric comorbidities. Mol Neurobiol 2014:49:1532-9.

22 van Eersel J, Ke YD, Liu X, et al. Sodium selenate mitigates tau pathology, neurodegeneration, and functional deficits in Alzheimer's disease models. Proc Natl Acad Sci U S A 2010;107:13888-93.

23 Cardoso BR, Roberts BR, Malpas CB, et al. Supranutritional sodium selenate supplementation delivers selenium to the central nervous system: results from a randomized controlled pilot trial in Alzheimer's disease. Neurotherapeutics 2019;16:192-202.

24 Rascovsky K, Hodges JR, Knopman D, et al. Sensitivity of revised diagnostic criteria for the behavioural variant of frontotemporal dementia. Brain 2011;134:2456-77.

25 Moroney JT, Bagiella E, Desmond DW, et al. Meta-analysis of the Hachinski ischemic score in pathologically verified dementias. Neurology 1997;49:1096-105.

26 Smith SM, De Stefano N, Jenkinson M, et al. Normalized accurate measurement of longitudinal brain change. $J$ Comput Assist Tomogr 2001;25:466-75.

27 Smith SM, Zhang Y, Jenkinson M, et al. Accurate, robust, and automated longitudinal and cross-sectional brain change analysis. Neuroimage 2002;17:479-89.

28 Hsieh S, Schubert S, Hoon C, et al. Validation of the Addenbrooke's cognitive examination III in frontotemporal dementia and Alzheimer's disease. Dement Geriatr Cogn Disord 2013;36:242-50.

29 Kramer JH, Mungas D, Possin KL, et al. NIH examiner: conceptualization and development of an executive function battery. $J$ Int Neuropsychol Soc 2014;20:11-19.

30 Burgess PW, Shallice T. Hayling and Brixton tests. Pearson, 1997.

31 Spreen O, Strauss E. A compendium of neuropsychological tests: administration, norms and commentary. 2nd ed. New York: Oxford University Press, 1998.

32 Benton AL, Hamsher K. Multilingual aphasia examination: manaul of instruction. lowa City: University of lowa, 1976.

33 Tombaugh TN. Trail making test a and B: normative data stratified by age and education. Arch Clin Neuropsychol 2004;19:203-14.

34 Wechsler D. Wechsler adult intelligence scale (WAIS-IV). Vol. 22. 4th edn. San Antonio, TX: NCS Pearson, 2008.

35 Savage S, Hsieh S, Leslie F, et al. Distinguishing subtypes in primary progressive aphasia: application of the Sydney language battery. Dement Geriatr Cogn Disord 2013;35:208-18.

36 Bertoux M, Delavest M, de Souza LC, et al. Social cognition and emotional assessment differentiates frontotemporal dementia from depression. J Neurol Neurosurg Psychiatry 2012;83:411-6.

37 Wear HJ, Wedderburn CJ, Mioshi E, et al. The Cambridge behavioural inventory revised. Dement Neuropsychol 2008;2:102-7.

38 Zarit SH, Reever KE, Bach-Peterson J. Relatives of the impaired elderly: correlates of feelings of burden. Gerontologist 1980;20:649-55

39 Chan D, Fox NC, Jenkins R, et al. Rates of global and regional cerebral atrophy in $\mathrm{AD}$ and frontotemporal dementia. Neurology 2001;57:1756-63. 